\title{
Research Workshop Collected Paper: Explorations in concepts and the visual arts
}

\author{
Graham Diprose \\ Researcher \& Author \\ Co-Chair EVA London \\ Independent \\ Redhill, Surrey \\ grahamdiprose@gmail.com
}

\author{
Frances Liddell \\ PhD Researcher \\ Institute for Cultural Practices \\ University of Manchester \\ Manchester, UK \\ frances.liddell@postgrad.manchester.ac.uk
}

\author{
Marco Pini \\ Independent Researcher \\ ggskips.com \\ Hackney \\ London, UK \\ marco@slowdance.co.uk
}

\author{
Sarah Vollmer \\ PhD Researcher \\ York University \\ Toronto, Ontario \\ Canada \\ vollmer@yorku.ca
}

\author{
Racelar Ho \\ PhD Researcher \\ York University \\ Toronto, Ontario \\ Canada \\ racelar@yorku.ca
}

\begin{abstract}
The EVA London Research Workshop is one of the more unique elements of our Conference that we have been keen to develop over many years. Often Postgraduate Students, at Masters or PhD level, or Unaffiliated Early Career Artists may feel excluded from prestigious conferences until their research is completed and they can submit a full paper proposal. However, over many years, EVA London has recognised the importance of offering an almost unique opportunity to submit projects that can be truly described as 'Work in Progress'. With an audience of International Academics and acknowledged experts in a field, the Research Workshop presentations have often lead to very positive interest and support, and sometimes to future collaborations. Others return to EVA London a year or two later with a completed piece of Research and a successful Full Conference Proposal. In previous years the presentations have been very popular with all of our delegates and we are delighted to include all of the presentations as part of our mainstream July 2021 Online conference. Although we will once again miss some of the informal networking opportunities, we have, as always, selected an exciting, ground breaking and quite eclectic group of RW delegates. We hope that by publishing their papers here, either grouped together, or individually published, some of you will be keen to contact our RW authors, to discuss and develop their ideas further.
\end{abstract}

Blockchain. Museums. NFTs. Mental Health. Chaos. Al. VR. Game Art. Haptics.

\section{INTRODUCTION}

\section{Graham Diprose, Co-Chair EVA London}

Our Research Workshop papers in the 2021 proceedings fall into two categories. Those who have arranged to have their paper published individually, perhaps as part of funding bid and those who are happy to be published as part of this group of three exciting short papers. The whole point of the EVA London Research Workshop, is to question and challenge accepted boundaries, which perhaps some other conferences, might tend to avoid. Our Artists in this 'Concepts and the Visual Arts' may well challenge some reader's pre- conceptions. I might venture to say that one of the reasons that EVA London remains a thriving and growing event, now in its $31^{\text {st }}$ year, has been it's continual ability to refresh and reinvent itself. We are delighted to attract more early career and unaffiliated artists to EVA London, than five or ten years ago. The Research Workshop is one of a number of ways that this goal has been achieved.

It is exceptionally rewarding for all of us involved in EVA London to see a 'Work in Progress' paper and presentation one year becoming a successful full conference paper the next. I hope that all the very talented researchers in this section will take note. With a conference called 'Electronic Visualisation in 
the Arts' almost inevitably the distinction and borders between Visual Art, New Concepts and Visual Technology will become blurred. Our RW combined paper will introduce you to three artists and curators who use clever technology and new concepts in order for them to explore, create and communicate their innovative visual ideas.

Whether you are an academic with digital arts students, or still studying, or are an unaffiliated artist, we are very keen each year to hear from you, if you might like to join our July 2022 EVA London Research Workshop.

\section{BLOCKCHAIN AND DIGITAL 'THINGNESS': EXPLORING THE OPPORTUNITIES AND RISKS FOR MUSEUMS}

\section{Frances Liddell, University of Manchester}

\subsection{Introduction: Blockchain and 'digital thingness'}

This paper seeks to analyse the opportunities and challenges of using blockchain, and specifically 'non-fungible tokens' (NFTs), in the museum context. Blockchain is a type of distributed ledger technology; it is essentially a database that records time-stamped and linked information, which is distributed across a peer-to-peer network of computers. The term is often associated with Bitcoin, however, blockchain is simply the infrastructure of this crypto-currency, and in recent years there has been considerable attention to exploring how the technology can challenge our understanding of ownership and authenticity in digital objects through the use of NFTs.

In brief, an NFT is a type of token stored through a blockchain that can represent assets such as artwork because these tokens are unique in value. This sense 'uniqueness' gives these tokens a sense of scarcity and authenticity that is not possible with digital images stored on the Internet due to the ubiquitous nature of the online object. Indeed, the use of an NFT enables a person to own a digital file and have a sense of control over it. In this respect, NFTs offer a 'new logic of thingness' to digital objects (Sacks et al. 2015; Zeilinger 2018, p.30), making them appear more 'real' and 'ontologically reified' (DuPont 2019, p.150).

2021 has been a year of increased NFT 'mania' with mass media starting to take note of these tokens (BBC 2021; Wintermeyer 2021; Luke, Shaw \& Stoilas 2021). This interest tends to focus on the use of NFTs as a monetary device for artists and collectors to buy and sell digital artwork online. However, this 'digital thingness' offers more than gaining an economic value and investigating alternative forms of value created through these tokens can prompt ideation of new use cases for NFTs in museums.

My project at the National Museum Liverpool (NML) sets out to explore these alternative forms of values by reproducing parts of the museum's digital collections as NFTs and handing these out to a group of participants. In doing so, I have been exploring to what extent these tokens can become a bind or a digital link between the museum and these participants and create a sense of shared ownership or guardian-ship over the token.

\subsection{The National Museums Liverpool Project}

This collaborative project with the NML took place in two stages: (1) ideation and (2) implementation. The first stage (ideation) invited a group of seven participants to the NML for a workshop in January 2020. In this workshop, these participants were asked to consider the idea of personal connection in objects and they were invited to choose one personal possession and one museum object from the NML based on this idea. These objects formed an online co-created exhibition called CryptoConnections, where each object is shown alongside the written summary from the participant that describes why they chose that object.

In the second stage (implementation) we used the digital objects in Crypto-Connections as the basis for the NFTs. Specifically, this second stage included the development of the 'Possession Gallery', a decentralised gallery connected to the Ethereum blockchain through a smart contract. Smart contracts are self-executing pieces of code that we use to interact with the Ethereum blockchain and, in this project, we used the smart contract to create an NFT of each object from Crypto-Connections and these were given out to each of the participants in the project.

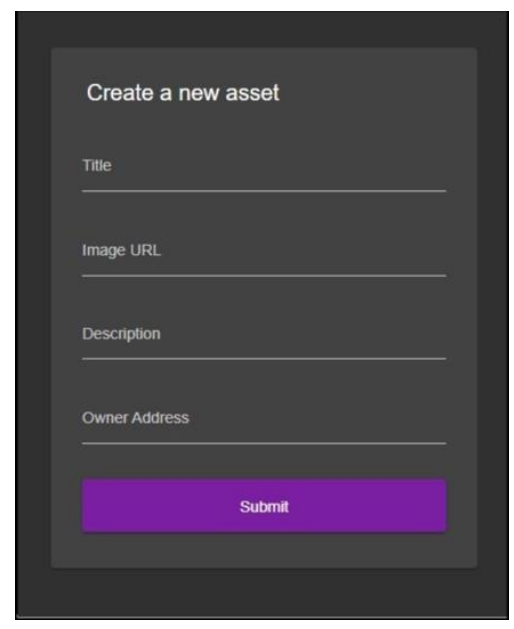

Figure 2.1: The admin page connected to the smart contract. The Possession Gallery. NML website. 
Figure 2.1 shows a screenshot of the admin page connected to this smart contract; the information needed to make each NFT includes a title, the image URL, the metadata/summary about the object, and the new owner's digital address. The smart contract combines this information together to create a token that permanently links all of this information together by storing it on the Ethereum blockchain as a transaction. Hence, the NFT represents a binding and permanent link between the participant and the object.

\subsection{Opportunities for 'digital thingness' in museums}

Due to the nature of blockchain and NFTs, this project raises questions around ownership, value, and authority. The digital thingness of an NFT has the potential to create a meaningful and identifiable digital object for the participant, which also embodies their personal experience about the depicted item. The technology also founds a new owner in the digital file since the token is owned by the participant rather than the museum. In turn, this challenges the museum's authority as the guardian of its collections because the token has formed a new digital museum object that is separate from the cultural institution. In this respect, the 'new logic of thingness' of an NFT offers an opportunity for the museum to inwardly challenge its own assumptions; both in terms of its use of digital museum objects as engaging devices and its control over them.

Therefore, NFTs offer a way of creating a 'shared authority' approach to digital collections. However, this idea is not a new concept in digital museum practices, for example, 'shared authority' has been examined in the context of Web 2.0 approaches and digital museum collections (Hazan 2007; Phillips 2014), but NFTs could provide a stronger emphasise on ownership and authenticity that could support a truly shared authority approach to digital museum collections.

In developing my research, I wish to consider how the application of blockchain or what a 'blockchain approach' might look like in practice in museums; are museums ready to relinquish such control? And, more importantly, are audiences ready to accept blockchain tokens as meaningful digital things?

\section{DELEUZIAN META-COGNITIVE THERAPY: THE OPTIMISTIC CHAOS OF HYDE PARK'S 'WINTER WONDERLAND'}

\section{Marco Pini, Independent Researcher}

\subsection{Introduction}

6 years ago, during my Philosophical Studies at Manchester University, I was diagnosed with Psychosis, Depression and Anxiety. Quite the meta-cognitive jolt, as I was, in parallel, studying the structures of thought Academically, and studying the structures of my own thoughts and mental health personally. Therapy, medication, mental health treatment creates a permanent awareness of what your mind is doing, of how you are thinking. Philosophy, learning, researching does the same. And across my recovery I have been in the process of learning about my mind, learning about how to better myself, how to interact and connect with people around me. I found that in writing about your journey through understanding the mind (which in itself is a large element of MBCT and CBT) you are accessing a rhizomatic web of connections, observing the jungle of cognitive distortions and through your health struggle, are forced to directly address meta-cognition. This high vantage point brings in relation things not previously perceived to be in contact with each other, a Schizophrenic cognition that Deleuze praises as 'living in intensive order' (Holland 2005).

\subsection{Recovery as a line of flight}

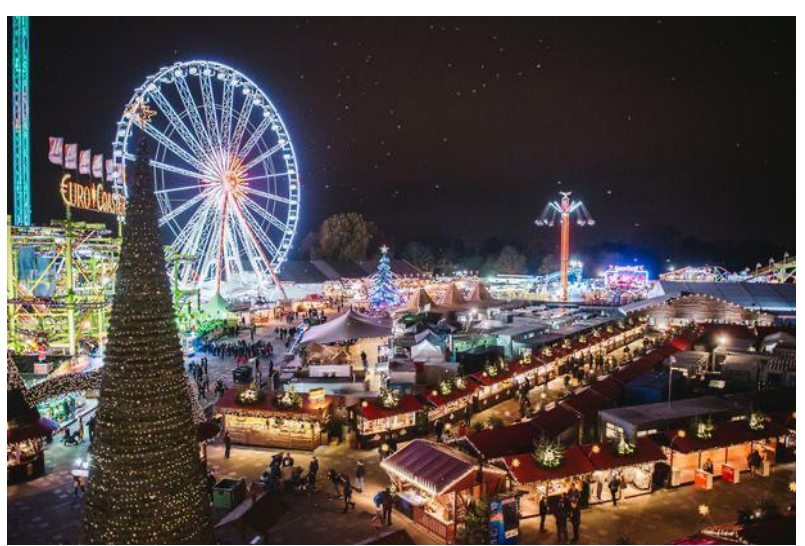

Figure 3.1: Winter Wonderland Hyde Park.

A process of recovery and change from one state to another (becoming) as a line of flight is what Deleuze and Guitarri refer to as '[a line] forever in the process of being drawn, toward a new acceptance, the opposite of renunciation or resignation - a new happiness.' In my recovery, I found two paths of positive acceleration that helped above all else; the philosophy of Deleuze and night-walking around Hyde Park's funfair attraction 'Winter Wonderland'. Bizarre to some, but for me, I 
wanted to understand the 'informal dimension' of the relationship, personal interest and movement between these two 'practical formations' that genuinely brought happiness to a serotonin deprived individual.

\subsection{Rhizomatic motivation and optimism}

Deleuze and Guiattari's theories of the world as a connected tapestry, a re-evaluation of the Freudian 'desire-as-lack' to a conception of positive desire, to a system of progression, which is both 'real and productive', takes its model from Nature. The Rhizome itself is the extension of roots and new pathways such as in the Strawberry Plant or resembled in the Network Theory of social animals such as Ants. The extension of these natural shoots, and their diagrammatic resemblance of cognitive, atomic and social structures is clearly optimistic to me. It is a chaotic, exponential drive to constant progress and change. Humans are active and motivated, not passive, and our discovery of 'the self' through folding and shifting, through extension allows refraction and alternative angles of understanding. The flight mirrors Entropy, or as Davies puts it, 'there are so many more ways to be untidy than to be tidy.' Winter Wonderland, for me, captures a topographical entropy described by Deleuze in Anti-Oedipus, it is a Maximalist heterotopia, a place containing many tones, crossroads of stiration and flow.

\subsection{Winter Wonderland}

Hyde Park's 'Winter Wonderland' is an artificial Christmas funfair, uniquely touristy and intense, a cosmic suggestion of the sublime. Uncanny and kitsch, the transient nature of this travelling Wonderland should detest most punters due to its 'gross' excess of the Capitalist Utopian machine. But to me, it is a glowing space of positivity in its structure. It has roots in a celebration of both the love of the Christmas period (an prime example of an organic vs. mechanical human invention) and a certain thoughtless appreciation taught by the practice of mindfulness, letting chaos be, observing the framework to manoeuvre with more ease. Walter Benjamin surmised that these funfares (then called Lunaparks) form a 'homeopathic, therapeutic intoxication or acceleration... from which the sick will draw strength.' This accelerationist reading, controversially stemming from fascist/futurist bodymachine utopias, can have a Positive charge. Benjamin's quasi-mystical acceptance that noticing the usually sickening (for the sick mind) products of capitalist acceleration, can, if accepted as simply a rhizomatic process of development, be a blissful representation of the working collective society, a cynicism of this reading that I have found to be detrimental to my view of the worlds creative melody. Winter Wonderland is also completely
Free, you can walk through it at any time of day, admire the glowing lights and chestnuts roasting, look upon the families enjoying the magic of the Christmas period, it is to me, the ultimate positive space. Deleuze claims in this sense that 'Everything is rational about Capitalism, except Capital.'

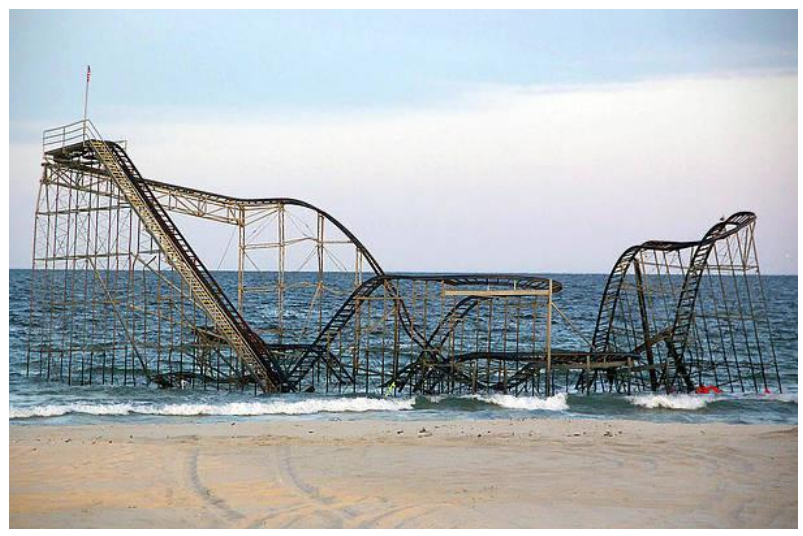

Figure 3.2 A rollercoaster that once sat on the Funtown Pier in Seaside Heights, N.J., rests in the ocean on Wednesday, Oct. 31, 2012 after the pier was washed away by super-storm Sandy that made landfall. (AP Photo/Julio Cortez) Julio Cortez, Associated Press.

\subsection{Roller Coasters, acceleration and tempo}

We can look at this ordered chaos in the spatiosymbolic structures of the rollercoasters towering over the Wonderland. The rollercoaster is a folded landscape, allowing multiple dimensions as well as an acceleration that cheats time by thrusting us forward against our control. It is a representation of a line of flight, a blurring of our tempo from the speed, becoming from point to point, ultimately ending up on the ground you started at. This is similar to the chaotic identification machine of Schizophrenics, outlined by Tuask - "the machine is of a mystical nature, 'the patients are able to give only vague hints of its construction" - levers, wheels, metal parts, steel... "all the discoveries of mankind, however are regarded as inadequate to explain the marvellous power of this machine, by which the patients themselves feel persecuted" (Tausk 1992).

The coaster, just one element of the Wonderland, explains my struggle, a knowledge of the parts that make up the system, but a lack of understanding of why you are on the ride. The Optimism in this is one exhibited in Mindfulness, CBT, Walking through a chaotic Wonderland and in the Philosophy of Deleuze, is the ability to sit back and simply observe the Maximalist Entropy. Allow it to be and appreciate the system as it is. Look at the sparkling lights, the screaming children, candyfloss and appreciate the beauty and complexity of chaos. 


\section{IRRATIONAL AND RATIONAL AVATAR: THE DIALOGUE BETWEEN POST-TECHNOLOGICAL HUMANS AND ARTIFICIAL INTELLIGENCE}

\section{Sarah Vollmer \& Racelar Ho, York University CA}

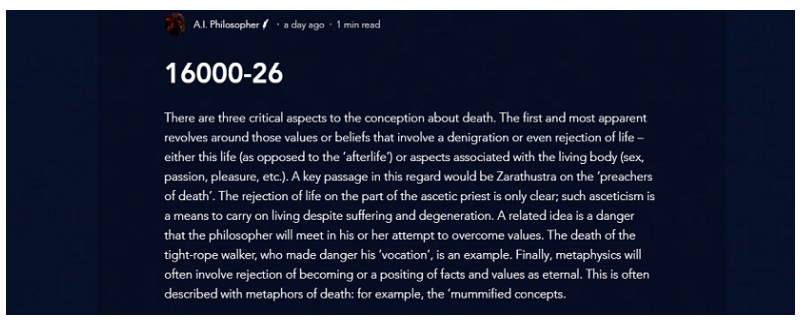

Figure 4.1: Our Al Philosopher posting through its interactive online blog.

\subsection{Conception of a generative dialogue}

We present here our Artificial Intelligence (AI) Philosopher and Virtual Reality (VR) Game-Art curator. Developed from a Zen-based ideology with inspiration from theories of animate and inanimate intelligence our cloud-based Al Philosopher (AIP) utilises Natural Language Processing (NLP) on texts describing Eastern and Western philosophies of nature, power, fear, love, space, and environment. In response to the growing climate crisis, our AIP applies this specialised training to environmental multimedia content uploaded to our virtual world-making framework where it is catalogued for interpretation. An evolving relationship to nature is cyclically crafted by this digital curation system through the AIP's poetic expressions and physical interpretations (e.g., Figure 4.1) of the accumulating submitted climate content where these interpretive landscapes and ecosystems are used to generate downloadable game content for an interactive VR game-artwork. Further, we establish the link between this climate dialogue and the Al curation that is transmitted to the VR world through the stimulation of a customised touch-based discourse.

\subsection{Irrational and rational avatars}

The hyper-intricate way things "mak[e] do" (de Certeau 2011) reveal innovative ideologies and extensive considerations of human being and machine relationships in deep space and time (Zielinski 2019). Our AIP is inspired by this very theory about the interdependence between the human intelligence versus mechanical intelligence, inanimate objects versus animate subjects, and machines versus non-machines. The collaborative "make do" framework of the AIP creates new philosophical content by inviting human audiences to share their minds with different formats and coestablish a new iteration training data for the neural network's intellectual growth. Combined, the irrationality of human intelligence and the absolute rationality of mechanical intelligence employ silent tactics to occupy and share the same time and space for weaving a post-technological legend (narrative). This project aims to identify the heterogeneous within natural intelligence and machine intelligence but also to proliferate this heterogeneous to make a human-machine subject that is trustable, rational, and irrational (Eagleton 1988).

\subsection{NLP and Al philosophers}

This new type of human-computer collaborative creation process introduced by this project is obscure and quiet, but also full of dramatic tension, just like the old typewriters used by humans in the past. This project's website and its real-time interaction (Figure 4.2) interface parallel the dichotomous comparison proposed by de Certeau: strategy versus tactic. The civilised world of humankind has "strategies" representing strength and power, while ordinary people write their legends through circuitous, cunning, and wise tricks or "tactics". Thus, we present an invitation for thought experiments on asking questions about the future world where this project solicits explorations of both construction models for a future civilised biology (human-machine collaboration) and of the profound relationship between art, science and technology (Jones and Hirsu 2019). AlPs do not intend to be constructed from a philosophical category made from nothing, on the contrary, they are built based on numerous human art texts and this human-only corpus connects with a collaborating human's current intelligent thoughts and brings the future to our present. It is here to discover novelty (new things) from old things, not to confirm the past again from new items.

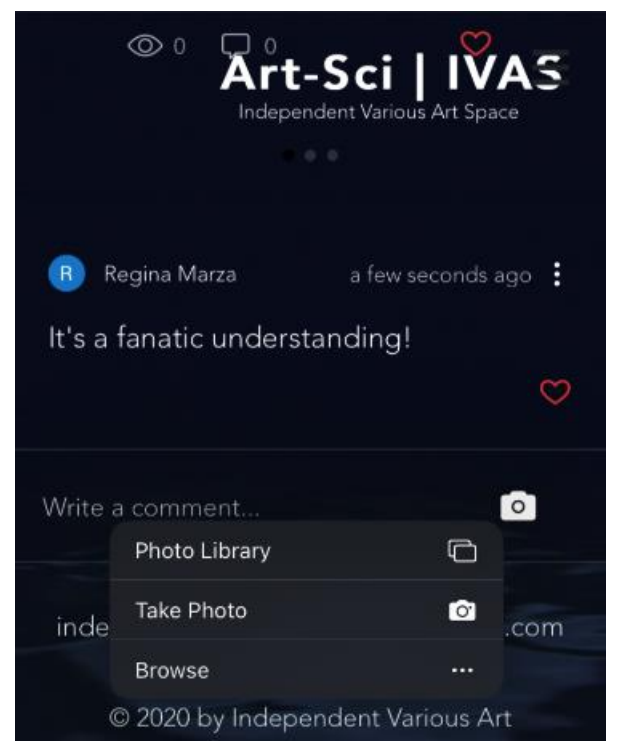

Figure 4.2: Mobile app interaction with our Al Philosopher. 


\subsection{Post-technological humans}

People experience a struggle every day: on one side are the rules and regulations for thinking and behaviour, which we can call consciousness from the spiritual perspective; on the other side is the free creation space of a single subject. The tension between the two is not an irreconcilable contradiction, on the contrary, the opposition is full of tension and complementary. Each party is indispensable to the other. The individual's freedom of will is not only blended with a world-defining idea, but the former is also dependent on the latter. Freedoms are, above all, an empirical concept. Free will-especially the intention to become aesthetic behaviour through fantasy-can only be realised through one's active thinking, judgment, creation, construction, struggle, and love. If it is a brief and concise sentence that summarises the nature of future plastic art from this perspective, this art is the ability to choose what one really wants-in a dialogue with another's will. The free will to change the world is a medium through which the subject can realise the self as the creator and consumer in the most extreme way.

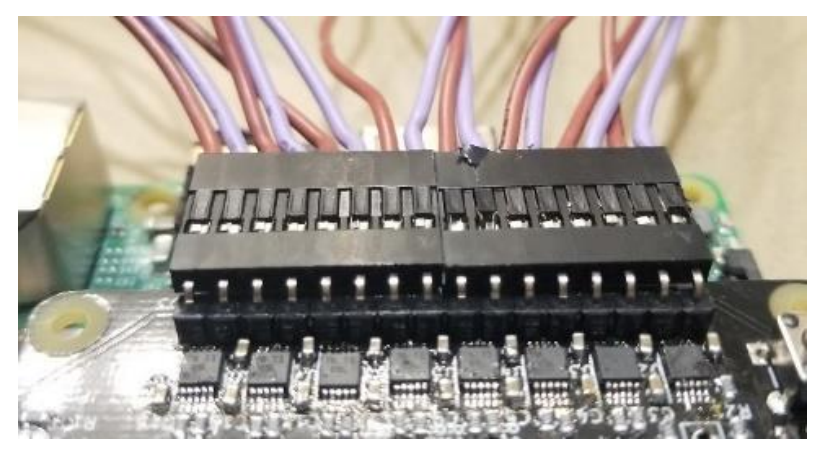

Figure 4.3: A set of custom haptics worn on the body.

\subsection{Tangible abstractions}

Now entering the second phase of development and testing, audiences can communicate to and with our philosophical curator-artist through text, images, and videos. Depending on the generative method selected, conversations with the AIP may receive different degrees of instant feedback through our website's two interactive modalities. Initial integration of custom haptic technology (Figure 4.3) and kinaesthetic experiences that evolve alongside, and are influenced by, the content evolution of the submitted user data, are in the process of evolving generative responses and reflect the suggested importance of making tangible these perceivably more abstract and displaced environmental issues.

The AIP thus demonstrates the intricacies of novel uses for dialogues both from and for humanmachine collaboration. By asking what we can create together, more-than-human worlds are made accessible through a fusion of the irrational and the rational where the post-technological and the artificial can thrive here and now to pursue viable futures in space and time.

\section{REFERENCES}

Benjamin, W., Jennings, M. and Bullock, M., n.d. Selected writings.

BBC (2021) 'NFT blockchain drives surge in digital art auctions', $B B C$ News. Available at: https://www.bbc.com/news/technology-56252738 (Accessed: 5 March 2021).

de Certeau, M. (2011) The Practice of Everyday Life. 3rd ed. University of California Press.

Colebrook, C., 2004. The Sense of Space: On the Specificity of Affect in Deleuze and Guattari. Postmodern Culture, 15(1).

Connolly, F., 2020. A New Happiness? Reading Literature with Deleuze and Guattari in 2020. Butler University.

Crypto-Connections.

https://www.liverpoolmuseums.org.uk/collections/cryptoc onnections (accessed 18 March 2021)

Davies, P., 2020. The demon in the machine. [Miejsce nieznane]: Penguin Books.

DuPont, Q. (2019) Cryptocurrencies and Blockchains. Cambridge \& Malden: Polity Press.

Eagleton, T. (1988) The Ideology of the Aesthetic. Poetics Today, 9(2), pp.327-38.

Fox, N., 2002. Refracting 'Health': Deleuze, Guattari and Body-Self. Health: An Interdisciplinary Journal for the Social Study of Health, IIIness and Medicine, 6(3), pp.347-363.

Hazan, S. (2007) 'A Crisis of Authority', in Theorizing Digital Cultural Heritage. Cambridge, Massachusetts \& London: MIT Press, pp.133-148.

Holland, E., 2005. Deleuze and Guattari's Anti-Oedipus. London: Routledge.

Horton, J., 2013. Mental Landscapes: Bazin, Deleuze, and Neorealism (Then and Now). Cinema Journal, 52(2), pp.23-45.

Jones, J. (ed) and Hirsu, L (ed). (2019) Rhetorical Machines: Writing, Code, and Computational Ethics. Tuscaloosa: University Alabama Press.

Luke, B., Shaw, A. and Stoilas, H. (2021) 'WTF are NFTs? Why crypto is dominating the art market'. (The Arts Newspaper). Available at: https://www.theartnewspaper.com/podcast/wtf-nfts (Accessed: 3 April 2021).

Noys, B., 2014. Malign velocities. Winchester, UK: Zero Books.

Phillips, L. (2014) 'The Role of Open Authority in a Collaborative Web', in Ridge, M. (ed.) Crowdsourcinig Our Cultural Heritage. Farnham, Surrey \& Burlington, USA: Ashgate, pp. 247-268. 
Roberts, M., 2006. Gilles Deleuze: psychiatry, subjectivity, and the passive synthesis of time. Nursing Philosophy, 7(4), pp.191-204.

Roh, J. et al. (2020). Hierarchical GPT with Congruent Transformers for Multi-Sentence Language Models. arxiv.org/pdf/2009.08636.pdf (15 March 2021).

Sacks, S. et al. (2015) 'Monegraph and the Status of the Art Object', Dis. Available at: http://dismagazine.com/discussion/73342/monegraphand-the-status-of-the-art-object/ (Accessed: 12 February 2021).

The Possession Gallery.

https://collectibles.liverpoolmuseums.org.uk/\#/ (Accessed 18 March 2021) N.B a digital wallet is needed to access this space.

Tausk, V., 1992. The influencing machine.

Van der Wielen, J., 2018. Living the intensive order: Common sense and schizophrenia in Deleuze and Guattari. Nursing Philosophy, 19(4), p.e12226.
Wintermeyer, L. (2021) 'Burned Banksy NFT Sets Art And Crypto Worlds Alight', Forbes. Available at: https://www.forbes.com/sites/lawrencewintermeyer/2021/ 03/03/burned-banksy-nft-sets-art-and-crypto-worldsalight/ (Accessed: 5 March 2021).

Zdebik, J., 2014. Deleuze and the diagram. London: Bloomsbury Academic.

Zielinski, S. (2006). Deep Time of the Media. The MIT Press, Cambridge, MA.

Zeilinger, M. (2018) 'Digital Art as "Monetised Graphics": Enforcing Intellectual Property on the Blockchain', Philosophy \& Technology, 31(1), pp. 15-41. doi: 10.1007/s13347-016-0243-1.

Zielinski, S. (2019) Variations on Media Thinking. Posthumanities. University of Minnesota Press, Minneapolis. 